\title{
Measurement in saliva from neurotypical adults of biomarkers pertinent to autism spectrum disorders
}

\begin{abstract}
Aim: Measure biomarkers pertinent to autism in saliva from humans. Materials \& methods: At 7:30 PM (reading instructions) and 8:30 PM (hearing instructions), neurotypical adults (6 M, $6 \mathrm{~F}$ ) each spat into tubes containing protease inhibitors. Cells were counted, samples aliquoted, frozen and thawed. Rationale was given for choice of biomarkers. ELISA: CD26, IL-12, carnitine, C4B, GSH, GSSG, MT2 , Testosterone, IFN- $\gamma$. Mass spectrometry: cystine, glutamine, glutamic acid, GABA, serotonin. Electrochemiluminescentimmunoassay: cortisol. Radioimmunoassay: Melatonin. Results: Cells averaged $2.16 \times 10^{6} / \mathrm{ml} . \mathrm{M}>\mathrm{F}$ : CD-26, C4B, MT-2. Testosterone, cortisol. Glutamine, glutamic acid, IFN- $\gamma$, melatonin and GSSG were measurable. Remaining biomarkers were measured in $<50 \%$ of samples. Concentrations were equal at both times. Conclusion: Saliva can be collected by literate individuals without added instruction. Ten biomarkers were measurable.
\end{abstract}

Keywords: autism • biomarkers $\bullet$ measurement $\bullet$ patient-specific therapy $\bullet$ saliva

Oxidative stress is central to the manifestation of autism [1]. This mechanism, along with immune glutamatergic dysfunction and pineal gland malfunction, provide 'unifying concepts' for a contributing cause of autism spectrum disorders (ASD) [2]. More than 70 biomarkers have been shown to be present in abnormal concentrations in autistic individuals, compared with age- and sex-matched controls [2]. However, it is doubtful if one single mechanism, one single unifying concept or one single biomarker will be adequate to diagnose and/or ascertain the degree of autism. This report documents our ability to measure biomarkers pertinent to autism in human saliva, and provides a basis for an objective measure of autism.

Jepson helped define the areas of the body in which autism is manifest: ubiquitous, gastrointestinal, immunologic, neurologic and toxicologic [3]. Nevertheless, the choice of biomarkers to define autism is very difficult. The rationale for the use of each of the biomarkers is given below. The referenced studies involved children with autism compared with age- and sex-matched controls.
Rationale of choice of biomarkers ( $\uparrow$ or $\downarrow$ denotes an increase or decrease found in ASD)

Ubiquitous

Carnitine

$\downarrow$ (serum). Concentrations of free and total carnitine were significantly reduced in a study of 145 autistic children between the ages of 2 and 13 years. Values for control individuals, age and/or gender specific, were used to create a normative range. The deficiency of carnitine, accompanied by slight elevations in lactate and significant elevations in alanine and ammonia, is a sign of mitochondrial dysfunction [4-10]. Recent studies suggest that dysregulation of carnitine metabolism may be important in nondysmorphic autism [5]. This study included 2904 individuals with simplex autism, 909 male probands from male-male sibling pairs, and over 8000 controls. Confirming carnitine's involvement with autism is the double-blind, placebo-controlled studies that used carnitine along with other treatments of mitochondrial disease, resulting in improved core and associated autistic symp-
Helen V Ratajczak*,1 \& Robert B Sothern ${ }^{2}$

${ }^{1}$ Edmond Enterprises, Danbury, CT 06810, USA

${ }^{2}$ College of Biological Sciences, University of Minnesota, St Paul, MN 55108, USA

*Author for correspondence:

Tel.: +1 2037786826

Fax: +1 2037486512

hratajcz@comcast.net 
toms [6]. Reduced levels of serum carnitine in autistic children have been shown to be related to gastrointestinal manifestations [7]. The autistic group included 78 males and 22 females aged between 3 and 10 years. Controls, apparently healthy, were also 78 males and 22 females, aged 3-10 years, unrelated to the autistic individuals. Carnitine is an essential co-factor in the utilization of fat reserves from body stores during fasting and stress. It plays a key role in the transport of long-chain fatty acids into the mitochondria where they undergo $\beta$-oxidation in energy production.

Glutamine, glutamic acid \& gamma amino butyric acid

$\downarrow$ (platelets, blood). Autoradiography showed an abnormality in the glutamine, glutamic acid \& gamma amino butyric acid (GABA) system in autism [8]. An imbalance in the GABA system could contribute to behavioral deficits in autism. Giving benzodiazepines, which are GABA receptor agonists, to some individuals with autism results in an adverse aggressive response [9]. The serotonergic system is intimately interconnected with GABAergic and glutamatergic neurons throughout the brain. Serotonin, a precursor of melatonin, is converted to $\mathrm{N}$-acetylserotonin in the pineal gland by the rate-limiting enzyme arylalkylamine $\mathrm{N}$-acetyltransferase (AA-NAT), followed by the conversion of $\mathrm{N}$-acetylserotonin to melatonin by acetylserotonin methyltransferase. There is a relationship among glutamine, GABA and social cognition in autism [10].

\section{Gastrointestinal system}

Dipeptidyl peptidase (DPP IV)/CD26

$\downarrow$ or absent in autistic individuals (urine). DPP IV is the only known enzyme to break down casomorphine. This marker is known as CD26 on T-lymphocytes. The absence of CD26 could explain the opioid-like casomorphine found to be elevated in urine from autistic patients [11]. There are alterations in plasma DPP IV in autistic individuals [12]. In addition, there is influence of candidate polymorphisms on the DPP IV and $\mu$-opioid receptor genes expression in the aspect of the $\beta$-casomorphin-7 modulation functions in autism [13]. There is controversy about urinary opioid peptides, with MALDI-TOF confirmation of absence of these peptides in children with autism. However, HPLC showed peaks in locations at which opioid peptides might be expected to be found [14]. The main idea of the upload-excess theory suggests that excessive levels of incompletely metabolized peptides from foods that contain proteins, gluten and casein pass through the intestinal and blood-brain barriers into the brain, where they directly regulate transmission in all the main neurotransmission systems or form ligands for peptidase enzymes that would normally hydrolyze naturally-occurring opioid peptides [15].

\section{Immunologic system}

\section{Complement component C4B}

$\downarrow$ (blood) [16]. The deficiency of C4B in the blood correlates with the complement $C 4 B$ gene null allele being more frequent in individuals with autism [17]. $C 4 B$ is necessary in the activation of the classical complement pathway that results in lysis [18]. With deficient C4B, the immune system is partially compromised. The link of C4B null allele to autism and to a family history of autoimmunity is described [19].

\section{IFN- $\gamma \&$ IL-12}

$\uparrow$ (plasma) [20,21]. IFN- $\gamma$ and IL-12 regulate the function of immunoregulatory $\mathrm{T}$ cells, especially $\mathrm{CD} 4^{+} \mathrm{T}$ helper cells. The increase of these two biomarkers may suggest a defect of immunoregulatory $\mathrm{T}$ cells in autism. In addition, the increase may be relevant to autoimmunity in autism because IFN- $\gamma$ is a cytokine of Th-1 cells, and antigenic stimulation of Th-1 cells has been implicated in the autoimmune pathogenesis of autism. IL-12 selectively stimulates Th-1 cells and initiates pathogenesis of organ-specific autoimmune diseases. The increased midgestational IFN- $\gamma$ and interleukins in women bearing a child with autism shows the influence of these biomarkers during gestation [20]. Serum IL-12 has been measured in autistic children [21]. IFN- $\gamma$ is proposed as a biomarker in autism [22].

\section{Neurologic system Cortisol}

$\downarrow$ (serum) [23]. Decreased cortisol in serum suggests an abnormality in the hypothalamo-pituitary-adrenal axis in individuals with autism. Cortisol has an important role in proper emotional development and functioning. Cortisol is considered to be a marker (prominent and consistent) rhythm in normal individuals, with peak production in the morning [24], but shows an aberrant circadian rhythm in autistic individuals. In addition, autistic children and their neurotypical controls both showed increased salivary cortisol in anticipation of re-exposure to a perceived stressor [25]. This circadian study compared cortisol levels at different times of day. Evening values for children with autism showed a gradual decrease over the course of sampling in the morning and were consistently elevated in comparison with the neurotypical group in the evening. Autistic individuals show clear dysregulation of the circadian rhythm in autism. Autistic children between the ages of 3 and 10 years showed increased serum cortisol response to stress compared 
with neurotypical controls [26]. In a study of autistic children between 7 and 12 years of age, low-functioning autistic children had higher mean salivary cortisol levels than the high-functioning autistic group, which did not differ from neurotypical control children [27]. Adolescence is a period of great physiological, psychological and social change. During adolescence, there are heightened diurnal basal cortisol levels in addition to higher cortisol in response to perceived stressors. It is a time of increased vulnerability in people already prone to enhanced physiological arousal and poor adaption to change, such as autism. Adolescents with autism show more variable diurnal regulation and increased response to stress [28].

\section{Melatonin \\ $\downarrow$ (plasma) [29]. Melatonin is produced at night in the dark by the pineal gland and is a key regulator of circa- dian and seasonal rhythms. The acetylserotonin methyl- transferase gene, encoding the last enzyme of melatonin synthesis, is deleted in some individuals with ASD. Mel- atonin has a crucial role in human cognition and behav- ior. In autistic individuals, melatonin has a dampened circadian rhythm [29]. In addition, the amount of mela- tonin is decreased in individuals with autism [30]. There is a feed-sidewards interaction among the various hor- mone-producing glands [31]. When one hormone is out of synchronization, all the other hormones are affected. Melatonin has been shown to be beneficial in treatment of children with developmental disabilities [32]. In addi- tion, others describe melatonin as a treatment for sleep problems in children with autism [33].}

\section{Serotonin}

$\uparrow$ (platelets, brain, blood, urine) $\downarrow$ (brain, cerebral spinal fluid). Platelet hyperserotonemia is generally considered the most robust and well-replicated biological finding in autism [34]. The relative concentration of serotonin may be high or low in autistic individuals compared with controls, but different in different parts of the brain in addition to different parts of the body. The differences in serotonin levels in autistic individuals have been documented [35,36]. It is important to note that in some individuals with autism, serotonin is low in the cerebral spinal fluid but elevated in blood and platelets. In addition, pregnancy is a critical time in the life of a human, with accompanying symptoms such as hypertension, gestational diabetes and depression having significant consequences in the fetus. During such symptoms, cortisol is increased in the mother, and plays an important role in causing dysregulation of serotonergic signaling through elevating the serotonin reuptake transporter production in the developing brain [37]. Cortisol, serotonin and testosterone are key regulators of social aggression [38]. Serotonin is a precursor of melatonin.

\section{Testosterone}

$\uparrow$ (serum) $\uparrow$ (prenatal) A study involving 92 male and female adult subjects, confirmed a positive correlation between salivary testosterone and autism [39]. The effect of testosterone on autism begins in the fetus. Studies of amniotic testosterone in humans suggest that fetal testosterone is related to specific sexually dimorphic aspects of cognition and behavior. It is suggested that autism may be an extreme manifestation of some male-typical traits, especially cognition and neuroanatomy. Influence of elevated fetal steroidogenic activity is associated with autism [40]. Concentrations of progesterone, 17a-hydroxy-progesterone, androstenedione and testosterone (sex steroids) and cortisol were positively associated with each other and were elevated in 128 amniotic fluid samples of males born between 1993 and 1999 who later were diagnosed with autism, Asperger syndrome or pervasive developmental disorder not otherwise specified compared with typically developing controls. This is the first direct evidence of fetal steroidogenic activity in autism. Studies of amniotic testosterone in humans suggest that fetal testosterone is related to specific (but not all) sexually dimorphic aspects of cognition and behavior [41]. The extreme male brain theory of autism is proposed by Baron-Cohen, linking autism with the male trait of systemizing as opposed to the female trait of empathizing [42]. It has been suggested that this gendering of certain capacities or aptitudes in the human population is unpersuasive, and could have serious consequences for treatment and services for girls and women on the autism spectrum [43]. An extreme female brain theory of psychosis in adults with autism with or without co-morbid psychosis is described, concluding that the bias for empathizing over systemizing may be linked with the presence of psychosis in people with autism. In women with autism and psychosis the link between mania/hypomania and an empathizing bias was greater than in men with autism [44]. There is a significant negative correlation between testosterone and whole-blood serotonin in autistic individuals [45]. The transsulfuration and androgen pathways interact, stemming from the fact that a critical regulatory step in the androgen pathway involves the metabolite dehydroepiandrosterone (DHEA). DHEA is a key initial regulatory metabolite in the androgen synthesis pathway, which can be converted down the androgen pathway toward testosterone or toward the normally favored storage molecule DHEA-sulfate. Marked elevation of adrenal steroids, including DHEA, were measured in saliva of prepubertal autistic children [46]. 
Toxicologic system

Cysteine

$\downarrow$ (plasma) [47]. Cysteine, an essential amino acid, is decreased in autistic children (who also have a decrease in methionine and S-adenosylmethionine-regulated cystathionine-gamma-synthase activity), premature or newborn infants, and subjects stressed by disease. Cysteine is the rate-limiting amino acid for glutathione synthesis [48]. There is a possible cyclical interaction between the methionine cycle-transsulfuration and androgen pathways in some children with autism. Significantly increased serum total testosterone was correlated with a significant decrease in plasma cysteine, reduced glutathione, methionine, cystathionine and homocysteine [49]. Because cysteine contains the thiol group ( $\mathrm{SH})$, it is the most chemically reactive natural amino acid found in cells. Protein thiols represent a larger active redox pool than glutathione, and are thus likely to be directly involved in the cellular defense against oxidative stress [50]. In extracellular plasma, the cysteine/cystine (thiol/disulfide) redox couple provides the redox environment for circulating immune cells. Also, in the plasma, cysteine redox ratios were decreased in autistic children [51].

\section{Glutathione}

$\downarrow$ (plasma) [47]. Glutathione (GSH) (reduced or total) is a key component of the methionine cycle and is the predominant low-molecular-weight thiol in animal cells [48]. GSH redox ratios were decreased in autistic children [51]. GSH plays important roles in antioxidant defense, nutrient metabolism and regulation of cellular events, including gene expression, DNA and protein synthesis, cell proliferation and apoptosis, signal transduction, cytokine production, immune response and protein glutathionylation. GSH deficiency contributes to oxidative stress, which plays a key role in aging, and the pathogenesis of many diseases, including cancer, heart attack, stroke, diabetes and autism. The GSH/GSSG (thiol/disulfide) redox couple is the major mechanism for maintaining the intracellular microenvironment in a highly reduced state needed for detoxification. The cysteine/cystine redox potential has been shown to be more oxidized than the intracellular GSH/GSSG, and is regulated independently [51].

\section{Oxidized glutathione (GSSG)}

$\uparrow$ (plasma) [47]. The consistent decrease in cysteine and GSH concentrations and GSH/GSSG in autistic children suggests an increased vulnerability to oxidative stress. GSH/GSSG is the most important redox couple and plays a crucial role in antioxidant defense, nutrient metabolism and the regulation of pathways essential for whole body homeostasis [48].
Metallothionein (MT-2)

$\downarrow$ (blood, urine) [52]. Blood and urine from a total of 503 patients with autism, Asperger's syndrome or atypical autism were compared with those from neurotypical controls in analysis of heavy metals (it should be noted that [52] is from a meeting, and the data were not peer-reviewed). The $\mathrm{Cu} / \mathrm{Zn}$ ratio was severely elevated in $85 \%$ of the patients. In addition, 99\% showed evidence of a metal-metabolism disorder, suggesting a problem with metallothionein. Metallothionein is intimately involved in the development and function of the immune response, development of neurons and in detoxification of heavy metals. These functions are involved in many of the classic symptoms of autism, including gastrointestinal tract problems, an enhanced sensitivity to toxic metals and abnormal behaviors. A report of altered heavy metals and transketolase in autistic spectrum disorder documents significant change in deposition of arsenic, mercury, copper and iron in autistic children [53]. A review of the neuroprotective and neuroregenerative properties of metallothioneins is given [54].

\section{Hypothesis}

We propose that a composite of biomarkers may be able to measure autism and define its severity. Longitudinal analysis of the array will be used to guide therapy and measure the effect of therapies. Some evidence that this approach might be successful is given by the improvement in symptoms of individuals with autism when treated with melatonin [55]. Also, James et al. [47] showed that giving those biomarkers to individuals with a deficit in the same biomarkers helped autistic individuals. For example, every component of the methionine cycle was shown to be in aberrant concentration in autistic subjects compared with control individuals. Plasma concentrations of methionine, S-adenosylmethionine (SAM) and homocysteine were significantly lower and S-adenosylhomocysteine (SAH) and adenosine concentrations were significantly higher than those in control children, while SAM:SAH was almost $50 \%$ lower in autistic children. James's nutritional intervention trial (oral supplementation of folinic acid and betaine) improved the concentrations of methionine, SAM, homocysteine, cystathionine, cysteine and total GSH (tGSH) and increased SAM:SAH and tGSH:GSSG. In addition, the high SAH and adenosine seen at baseline decreased. A second intervention added an injectable form of methylcobalamin (Vitamin B-12) to the above regimen. This further decreased those concentrations of adenosine and GSSG to normal levels, and increased the concentrations of those biomarkers that were too low (methionine, cyste- 
ine, $\mathrm{tGSH}$, SAM:SAH and $\mathrm{tGSH}$ :GSSG) to normal concentrations. A list of parent ratings of behavioral effects of biomedical interventions in autistic individuals has been recorded [55]. Examples include 54\% of 2738 autistic individuals improved when treated with zinc, $66 \%$ of 1687 improved when treated with melatonin and $72 \%$ of 899 improved when treated with methyl B12. These studies recorded effects of treatment of autistic individuals. Control individuals were not treated.

\section{Current study}

In order to probe into those biomarkers identified with autism, we studied at least one biomarker from each of the systems of the body in which autism is manifest: gastrointestinal, immunologic, neurologic, toxicologic and ubiquitous (e.g., those biomarkers involved in the entire body) [3]. These biomarkers are also involved in unifying concepts of autism: increased vulnerability to oxidative stress, immune glutamatergic dysfunction and pineal gland malfunction [2].

The body fluid chosen for the measurement of biomarkers was saliva. Biomarkers have been identified in saliva from autistic individuals [56-58]. Collecting either blood or urine is more stressful to the subject than collection of saliva [58]. Saliva can be used as an adjunctive diagnostic aide for measurement of oxidative stress in autism. Also, salivary glutathione is a powerful predictor of autism spectrum disorder [59]. One of the biomarkers shown to be lower in concentration in individuals with autism is cortisol [23]. It should be noted that stress causes the amount of cortisol to be produced at a greater concentration [60]. This phenomenon is also present in autistic children, demonstrated by production of increased cortisol in anticipation of re-exposure to a perceived stressor $[25,61]$. As previously mentioned, it is known that alteration in production of one hormone affects the production of other hormones, caused by a feedsidewards interaction [31]. Therefore, when choosing a body fluid for measurement of biomarkers, it is important to avoid stress as much as possible.

Our initial research used saliva from neurotypical adults to measure 16 biomarkers pertinent to autism. Assays included ELISA and MS. Because these methods were not sufficiently sensitive for some of the biomarkers, other more sensitive methods were utilized (see 'Materials \& methods' section).

\section{Authorization}

This study was authorized by the New England Independent Review Board, Newton, MA, USA and was performed under the guidance of Ms Ann McLellan, Quality Assurance Officer, and in accordance with the ethical standards laid down in the 1964 Declaration of Helsinki and its later amendments regarding ethical principles for medical research involving human subjects. All persons gave their informed consent in writing prior to their inclusion in the study.

\section{Materials \& methods}

\section{Subjects}

Twelve subjects (six men and six women) were screened prior to admission to the study, and all met the inclusion/exclusion criteria: between 21 and 80 years of age and free of neurological disorder or pathogenic disease, with no oral problems that would interfere with salivary sampling. No female was pregnant or lactating. Males included three Caucasians, one African-American and two Asians; females included four Caucasians and two Asians.

\section{Sample collection}

The twelve neurotypical healthy adults gave samples of saliva at two separate times (7:30 and 8:30 PM, or 19:30 h and 20:30 h, military time) in order to ascertain if production of biomarkers would be similar after subjects read instructions alone compared with having been given instruction by the principal investigator. In addition, at these times the concentration of two of the chosen markers for which the circadian rhythm has been established, cortisol and melatonin, is usually lowest within a period of $24 \mathrm{~h}$ in blood, urine and saliva [62,63]. If these biomarkers can be measured, it is an indication that greater levels produced at different times within the 24-h day will also be able to be measured. Also, similar measurements an hour apart provide proof that approximately equal concentrations are present within that span of time, allowing flexibility in timing of collection of specimens for future chronobiological studies.

Each subject documented that he/she refrained from eating, drinking, brushing or flossing his/her teeth for $1 \mathrm{~h}$ prior to the first collection at 19:30 h. Then he/she washed their hands with soap (Ivory Liquid) and water, and sat down. Each drank 8 ounces of tap water from a styrofoam cup. Then he/she spit into a $50 \mathrm{ml}$ polypropylene conical centrifuge tube containing $50 \mu \mathrm{L}$ of a protease inhibitor (Cocktail, EDTA-Free, Thermo Scientific Halt Protease Inhibitor Cocktails, Catalog \#78425) until at least $5 \mathrm{ml}$ (indicated by a black mark on the outside of the tube) were collected. This procedure was repeated by each subject an hour later at 20:30 h for a total collection of 24 saliva samples. Each sample of saliva was gently mixed, and the cells in each sample were counted prior to making 10 aliquots of $0.5 \mathrm{ml}$ each. All tubes 
used were polypropylene. Aliquots and the original tube were then frozen at $-80^{\circ} \mathrm{C}$.

\section{Laboratory analyses}

Cell counts

Cells were counted with the aid of a Cellometer Cell Counter (Nexcelom Bioscience).

\section{Biomarkers}

Each assay utilized an aliquot of each sample the first time it was thawed. Sixteen biomarkers were analyzed: glutamine, glutamic acid, CD-26, C4B, IFN- $\gamma$, MT-2, testosterone, IL-12, Carnitine, GSH, GSSG, cystine, GABA, serotonin, cortisol and melatonin.

ELISAs, conducted in triplicate (total of 36 replicates at each time point), were used to measure the biomarkers listed in Table 1. Only one ELISA, that for CD26, was designed for measuring quantities of the biomarker in saliva. The other kits were designed for measuring the biomarkers in plasma, serum or urine. Because concentrations of biomarkers are less in saliva, in general, than in serum or plasma $[62,63]$, the standard curve was extended for four additional serial dilutions to enable measurement of low concentrations. The manuals accompanying the ELISA kits gave information for the minimum detectable dose (MDD, the lowest dose that could be distinguished from zero) and the lowest limit of quantitation (LLOQ, the lowest point in the standard curve).

In Table 2, the MDD is that from the manual accompanying each ELISA kit, while the LLOQ is the lower limit of quantitation determined by extension of the standard curve by four serial dilutions.

\section{Mass spectrometry (Shimadzu LC System) \\ Amino acids}

The aTRAQ ${ }^{\mathrm{TM}}$ Reagent Kit 50 Assay (AB Sciex Cata$\log \#$ 4442668) was used to measure cystine, glutamine, glutamic acid and GABA $(\mu \mathrm{M} / \mathrm{ml})$. These assays were run in duplicate, with a total of 24 replicates at each time point.

\section{Hormones}

Serotonin, cortisol, melatonin and testosterone were measured from one sample of each specimen, with a total of 12 measurements at each time point. Analytes used are listed in Table 3.

\section{More sensitive methods}

Because the original methods were not sensitive enough to measure some of the biomarkers in the majority of the samples, we had another laboratory use more sensitive methods.

NeuroScience, aka The Pharmasan Lab Inc. (WI, USA), measured three of the hormones by more sensitive methods: cortisol (Electrochemiluminescent Immuno Assay), melatonin (Radio Immuno Assay) and testosterone (Enzyme-linked Immunosorbent Assay) (see Table 4).

\section{Glutathione forms}

Glutathione (GSH) rapidly oxidizes to GSSG and detection by ELISA was unsatisfactory.

\section{Results}

There were 12 samples at $19: 30$ and 12 samples at 20:30 collected for analysis of cells and the 16 biomarkers. Overall averages using timepoint mean values for each biomarker from males and females $(\mathrm{M}+\mathrm{F})$ at both collection times $(19: 30+20: 30)$, and averages from $M+F$ at 19:30 versus 20:30 and $M$ versus $F$ at 19:30 + 20:30 are summarized in Table 5. Note: if triplicate values were available, means were only calculated if two or more were valid numbers (i.e., $>0$ ), but when duplicates were available, if one sample was zero, the second sample was used as a valid number. $\mathrm{p}$-values from unpaired, 2-sided t-tests are listed, with any $\mathrm{p} \leq 0.05$ shown in bold.

\section{Cell numbers}

The mean \pm SE number of cells $\left(\times 10^{6} / \mathrm{ml}\right)$ for $\mathrm{M}+\mathrm{F}$ overall from 23 samples was $2.16 \pm 0.26$ (individual

\begin{tabular}{|llll|}
\hline $\begin{array}{l}\text { Table 1. Human ELISA kits used. } \\
\text { Variable (Abbreviation) }\end{array}$ & Kit & Catalog no. & Manufacturer $^{\dagger}$ \\
\hline Dipeptidyl peptidase IV/CD26 & Quantikine & DC260 & 1 \\
\hline IL-12 & Quantikine & D1200 & 1 \\
\hline Total carnitine & Cusabio Biotech Co., Ltd. & CSB-E13242h & 2 \\
\hline C4B & USCN Life Science Inc. & E91305Hu & 2 \\
\hline GSH & Cusabio Biotech Co., Ltd. & CSB-E09495h & 2 \\
\hline MT-2 & Cusabio Biotech Co., Ltd. & CSB-E13535h & 2 \\
\hline IFN- $\gamma$ & Quantikine & DIF50 & 1 \\
\hline GSSG & Cusabio Biotech Co., Ltd. & CSB-E13735h & 2 \\
\hline${ }^{+1: R \& D ~ S y s t e m s, ~ M i n n e a p o l i s ~ M N ~ 55413, ~ U S A ; ~ 2: ~ W u h a n, ~ H u b e i ~ P r o v i n c e ~ 430223, ~ P . R . ~ C h i n a ~}$ & \\
\hline
\end{tabular}




\begin{tabular}{|c|c|c|c|c|c|c|c|c|}
\hline \multirow[t]{2}{*}{ Biomarker } & \multirow[t]{2}{*}{ Units } & \multirow[t]{2}{*}{$n$ values } & \multirow[t]{2}{*}{ MDD } & \multicolumn{2}{|c|}{ Values >MDD } & \multirow[t]{2}{*}{ LLOQ } & \multicolumn{2}{|c|}{ Values > LLOQ } \\
\hline & & & & n & $\%$ & & $\mathbf{n}$ & $\%$ \\
\hline C4B & $\mathrm{ng} / \mathrm{ml}$ & 24 & 1.90 & 24 & 100 & 0.50 & 24 & 100 \\
\hline CD26 & $\mathrm{ng} / \mathrm{ml}$ & 23 & 0.016 & 23 & 100 & 0.018 & 23 & 100 \\
\hline MT-2 & $\mathrm{ng} / \mathrm{ml}$ & 23 & 0.80 & 23 & 100 & 0.19 & 23 & 100 \\
\hline GSSG & $\mathrm{pg} / \mathrm{ml}$ & 23 & 9.38 & 20 & 87 & 0.29 & 20 & 87 \\
\hline IFN- $\gamma$ & $\mathrm{pg} / \mathrm{ml}$ & 22 & 8.00 & 13 & 59 & 0.90 & 17 & 77 \\
\hline Carnitine & $\mathrm{nmol} / \mathrm{ml}$ & 23 & 0.78 & 8 & 35 & 0.10 & 11 & 48 \\
\hline GSH & $\mu \mathrm{g} / \mathrm{ml}$ & 23 & 0.20 & 1 & 4 & 0.049 & 2 & 9 \\
\hline IL-12 & $\mathrm{pg} / \mathrm{ml}$ & 23 & 5.00 & 0 & 0 & 0.40 & 2 & 9 \\
\hline
\end{tabular}

range: 0.28 to 4.40 ). There were no significant (n.s.) differences (Table 5).

\section{Biomarkers}

Ubiquitous system

Carnitine $(\mathrm{nmol} / \mathrm{ml})$ was measurable above the LLOQ in $11 / 23(48 \%)$ of the total samples ( 5 at $19: 30$ and 6 at 20:30, 8 for M, 3 for F). The overall mean was 28.5 \pm 18.1 (individual range: 0.60 to 201.0 ), $53.9 \pm 38.5$ at $19: 30$ versus $7.4 \pm 3.1$ at $20: 30$ (n.s.) and $37.7 \pm 24.4$ for $M$ versus $4.0 \pm 2.2$ for $F$ (n.s.).

Glutamine $(\mu \mathrm{M} / \mathrm{ml})$ was measurable above the LLOQ in $22 / 23(96 \%)$ of the total samples (11 at 19:30 and 11 at 20:30, 11 for M, 11 for F). The overall mean was $10.4 \pm 3.8$ (individual range: 1.5 to 89.2 ), $14.7 \pm 7.5$ at $19: 30$ versus $6.1 \pm 1.2$ at $20: 30$ (n.s.) and $15.4 \pm 7.5$ for $M$ versus $5.4 \pm 1.0$ for $F$ (n.s.).

Glutamic acid $(\mu \mathrm{M} / \mathrm{ml})$ was measurable above the LLOQ in $22 / 23(96 \%)$ of the total samples (11 at $19: 30$ and 11 at 20:30, 11 for M, 11 for F). The overall mean was $23.3 \pm 5.4$ (individual range: $4.2-124.1$ ), $28.4 \pm 10.1$ at $19: 30$ versus $18.2 \pm 3.7$ at $20: 30$ (n.s.) and $30.5 \pm 10.2$ for $M$ versus $16.1 \pm 2.7$ for $F$ (n.s.).

GABA $(\mu \mathrm{M} / \mathrm{ml})$ was measurable above the LLOQ in $11 / 23(48 \%)$ of the total samples (5 at $19: 30$ and 6 at 20:30, 8 for M, 3 for F). The overall mean was 2.40 \pm 1.05 (individual range: 0.39 to 12.53 ), $4.19 \pm 2.11$ at $19: 30$ versus $0.91 \pm 0.25$ at $20: 30$ (n.s.) and 2.80 \pm 1.42 for $M$ versus $1.35 \pm 0.70$ for $F$ (n.s.).

\section{Gastrointestinal system}

CD26 $(\mathrm{ng} / \mathrm{ml})$ was measurable above the LLOQ in $23 / 23(100 \%)$ of the total samples (11 at 19:30 and 12 at 20:30, 12 for M, 11 for F). The overall mean was $16.7 \pm 1.8$ (individual range: 3.7 to 42.7 ), $18.1 \pm 3.1$ at $19: 30$ versus $15.4 \pm 2.1$ at $20: 30$ (n.s.) and $21.4 \pm 2.5$ for $M$ versus $11.5 \pm 1.6$ for $F(p=0.004)$.

\section{Immunologic system}

C4B (ng/ml) was measurable above the LLOQ in $24 / 24(100 \%)$ of the total samples (12 at 19:30 and 12 at 20:30, 12 for M, 12 for F). The overall mean was $199 \pm 36$ (individual range: 42 to 601 ), $216 \pm 59$ at $19: 30$ versus $181 \pm 43$ at 20:30 (n.s.) and $274 \pm 64$ for $M$ versus $124 \pm 18$ for $F(p=0.033)$.

IFN- $\gamma(\mathrm{pg} / \mathrm{ml})$ was measurable above the LLOQ in $17 / 23(74 \%)$ of the total samples ( 8 at $19: 30$ and 9 at 20:30, 10 for M, 7 for F). The overall mean was

Table 3. Analytes for measurement of hormones by MS.

\begin{tabular}{|lll|}
\hline Analyte & Manufacturer & Catalog number \\
\hline Cortisol & Cerilliant, TX 78665, USA & C-106 \\
\hline Melatonin & Cerilliant, TX 78665, USA & M-095 \\
\hline Serotonin hydrochloride & Sigma Aldrich, MO 63178, USA & H9523 \\
\hline Testosterone & Cerilliant, TX 78665, USA & T-037 \\
\hline N-Acetyl-5-methoxytryptamine-d4 & CDN Isotopes, Pointe-Claire, & D-2952 \\
& Quebec H9R 1H1, Canada & \\
\hline Cortisol-d4 & Cerilliant, TX 78665, USA & C-113 \\
Testosterone-d3 & Cerilliant, TX 78665, USA & T-046 \\
\hline Human saliva & BioReclamation, NY 11590, USA & HMSALIVA \\
\hline
\end{tabular}


Table 4. Hormone analysis by more sensitive methods.

\begin{tabular}{llll}
\hline Hormone & Means of analysis & Reagent manufacturer & Limits of detection \\
Cortisol & Electrochemiluminescent immuno assay & Roche, IN 46256, USA & $0.36-30 \mathrm{ng} / \mathrm{ml}$ \\
\hline Melatonin & Radio immuno assay & IBL, MN 55432, USA & $1-300 \mathrm{pg} / \mathrm{ml}$ \\
Testosterone & Enzyme linked immunosorbent assay & DRG Diagnostics D-35039 Marburg, Germany & $7.1-4500 \mathrm{pg} / \mathrm{ml}$ \\
\hline
\end{tabular}

$27.0 \pm 4.5$ (individual range: 2.1 to 65.0 ), $27.8 \pm 6.5$ at $19: 30$ versus $26.3 \pm 6.6$ at $20: 30$ (n.s.) and $25.1 \pm 5.4$ for $M$ versus $29.7 \pm 8.2$ for $F$ (n.s,).

IL-12 (pg/ml) was measurable above the LLOQ in $3 / 23(13 \%)$ of the total samples (2 at 19:30 and 1 at 20:30, 1 for M, 2 for F). The overall mean was 0.53 \pm 0.16 (individual range: $0.23-0.80$ ), $0.68 \pm 0.12$ at $19: 30$ versus 0.23 at $20: 30$ and 0.80 for $M$ versus 0.40 \pm 0.17 for F. T-tests for differences between sexes or timepoints could not be computed.

\section{Neurologic system}

Cortisol $(\mathrm{ng} / \mathrm{ml})$ was measurable above the LLOQ in 24/24 (100\%) of the total samples (12 at 19:30 and 12 at 20:30, 12 for M, 12 for F). The overall mean was $1.29 \pm 0.23$ (individual range: $0.20-5.80$ ), $1.53 \pm 0.43$ at $19: 30$ versus $1.06 \pm 0.19$ at $20: 30$ (n.s.) and 1.77 \pm 0.42 for $M$ versus $0.82 \pm 0.08$ for $F(p=0.038)$.

Melatonin $(\mathrm{pg} / \mathrm{ml})$ was measurable above the LLOQ in 22/24 (92\%) of the total samples (12 at 19:30 and 10 at 20:30, 10 for $M, 12$ for F). The overall mean was $2.59 \pm 0.33$ (individual range: $1.00-6.90$ ), $2.93 \pm 0.54$ at $19: 30$ versus $2.18 \pm 0.31$ at $20: 30$ (n.s.) and 2.87 \pm 0.61 for $M$ versus $2.36 \pm 0.33$ for $F$ (n.s.).

Serotonin $(\mathrm{ng} / \mathrm{ml})$ was measurable above the LLOQ in $2 / 23(9 \%)$ of the total samples ( 1 at 19:30 and 1 at $20: 30,0$ for M, 2 for F). The overall mean was $3.63 \pm$ 0.39 (individual range: $3.24-4.02$ ) and 3.24 at 19:30 versus 4.02 at 20:30. T-tests for differences between sexes could not be computed.

Testosterone $(\mathrm{pg} / \mathrm{ml})$ was measurable above the LLOQ in $22 / 23(96 \%)$ of the total samples (11 at 19:30 and 11 at 20:30, 12 for M, 10 for F). The overall mean was $31.7 \pm 4.3$ (individual range: $8.8-76.5$ ), 30.5 \pm 5.8 at $19: 30$ versus $32.9 \pm 6.5$ at $20: 30$ (n.s.) and 42.0 \pm 5.7 for $M$ versus $19.4 \pm 3.8$ for $F(p=0.005)$.

\section{Toxicologic system}

Cystine $(\mu \mathrm{M} / \mathrm{ml})$ was below the LLOQ in all samples. $\mathrm{rGSH}(\mu \mathrm{g} / \mathrm{ml})$ was measurable above the LLOQ in $2 / 23(9 \%)$ of the total samples (2 at 19:30 and 0 at 20:30, 1 for $M, 1$ for F). The overall mean was $2.92 \pm$ 2.12 (individual range: 0.80 for $\mathrm{M}$ vs 5.04 for $\mathrm{F}$ ). T-tests for differences between sexes could not be computed.

GSSG $(\mathrm{pg} / \mathrm{ml})$ was measurable above the LLOQ in 20/23 (87\%) of the total samples (9 at 19:30 and 11 at 20:30, 11 for M, 9 for F). The overall mean was $263 \pm$
18 (individual range: 42 to 601 ), $295 \pm 24$ at 19:30 versus $236 \pm 24$ at 20:30 (n.s.) and $254 \pm 29$ for $M$ versus $273 \pm 21$ for $F$ (n.s.).

MT-2 $(\mathrm{ng} / \mathrm{ml})$ was measurable above the LLOQ in $23 / 23(100 \%)$ of the total samples (11 at 19:30 and 12 at 20:30, 12 for M, 11 for F). The overall mean was $106 \pm 13$ (individual range: $26-201$ ), $101 \pm 19$ at $19: 30$ versus $110 \pm 19$ at 20:30 (n.s.) and $137 \pm 17$ for $M$ versus $71 \pm 14$ for $F(p=0.009)$.

See Figure 1 for comparison of times of measurement and comparison of values for males versus females ( $\mathrm{M}$ vs F) for those biomarkers that could be measured in the majority of samples. Note that Figure 1 shows values from the ELISA assay for GSSG.

Comparisons are shown between sexes and between sampling times, with statistical differences if $\mathrm{p}<0.05$ shown with an asterisk. Significant differences between sexes were found for CD26, C4B, cortisol, testosterone and MT-2, and for each of these biomarkers, the males had significantly higher levels than females. In contrast, there were no significant differences between measurement times for all $\mathrm{M}+\mathrm{Fs}$ combined.

See Table 6 for comparisons of current results with those of neurotypical adults published in the literature. When converted to the same units, our data show the saliva to contain less than the literature's reports of the same biomarkers in plasma or serum, with the exception of four biomarkers (GABA, testosterone, GSH, MT-2), which were more prevalent in saliva. Although our data are limited to samples from 12 individuals tested on two occasions, the data show that some biomarkers are greater in saliva than blood, and support saliva as the body fluid of choice for measuring biomarkers pertinent to autism.

\section{Discussion}

When designing this study, a major consideration was to keep the saliva collection process as simple as possible in anticipation of collecting from autistic subjects in a future study. Although there are many collection devices on the market, the most noninvasive and stressfree method is simply to have the subject spit into a large tube. In addition, the tubes used for collection and storage of the saliva are made of polypropylene in an effort to avoid any adherence of the saliva components to the storage vessel, as well as any possibility of breakage (if glass were used). 


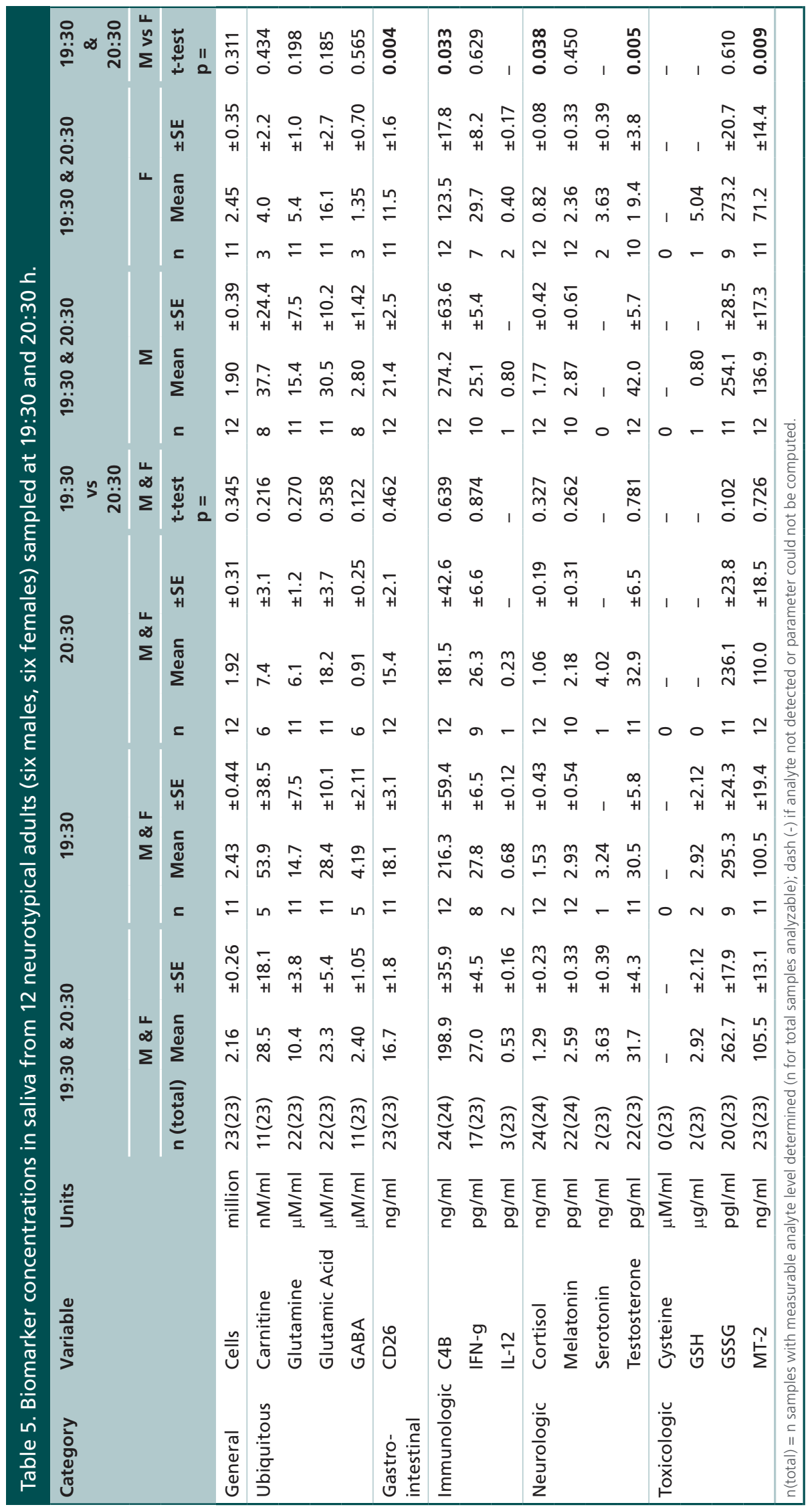



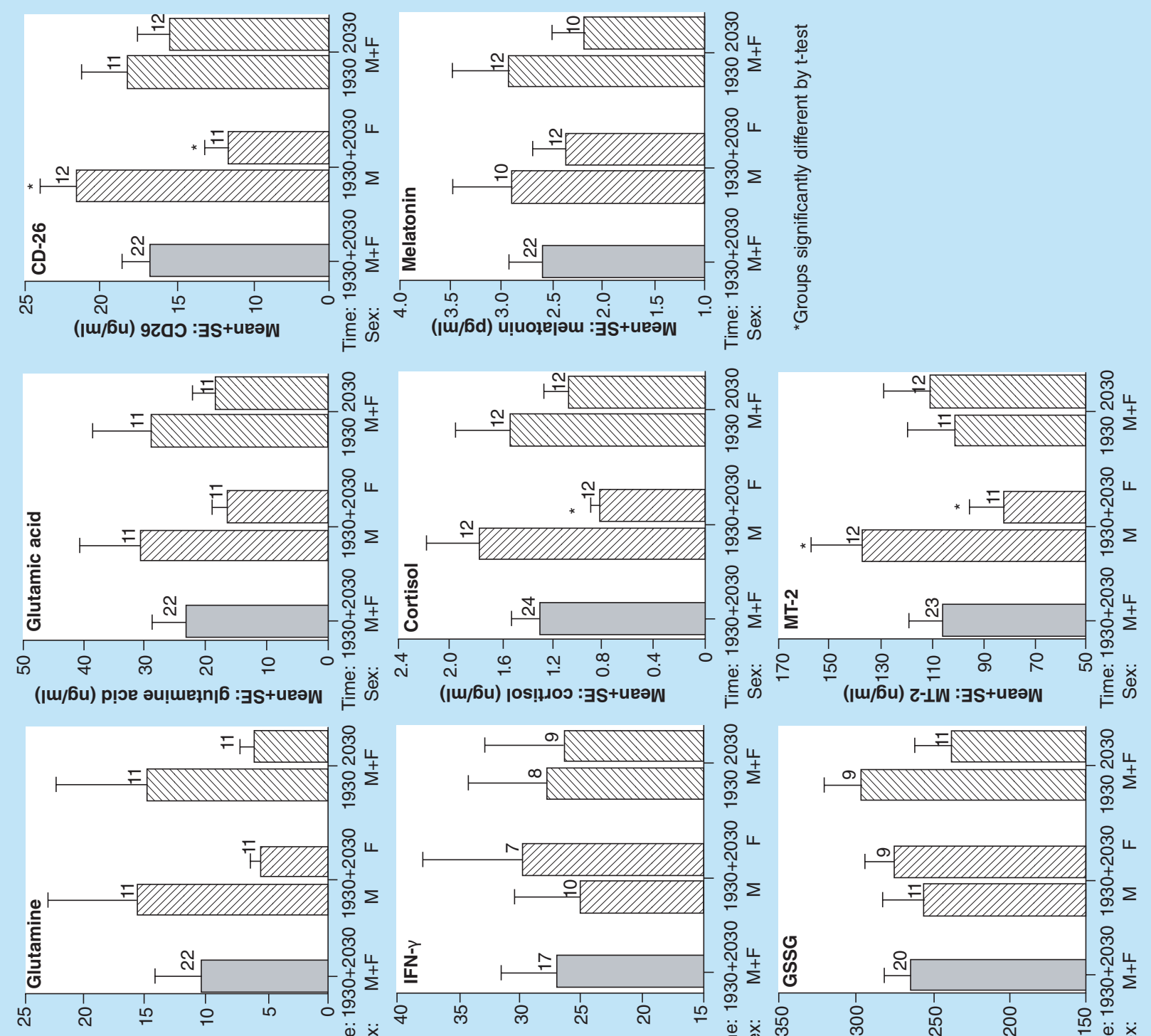

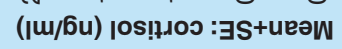

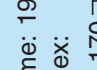

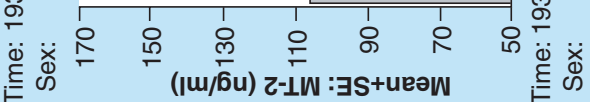
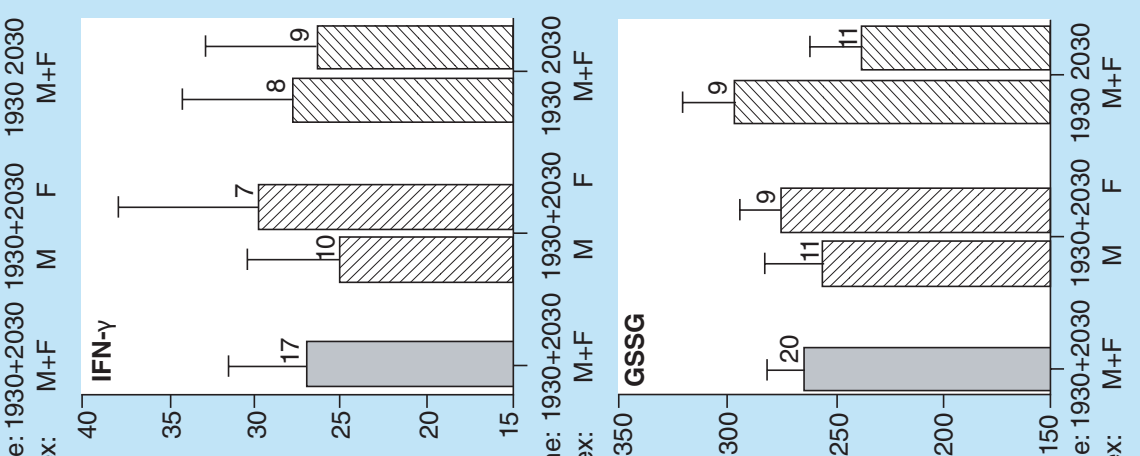

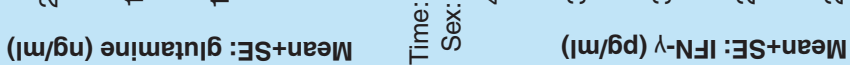

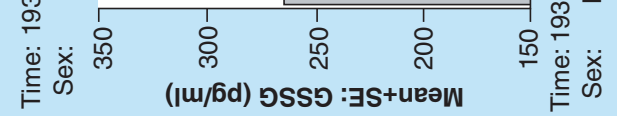
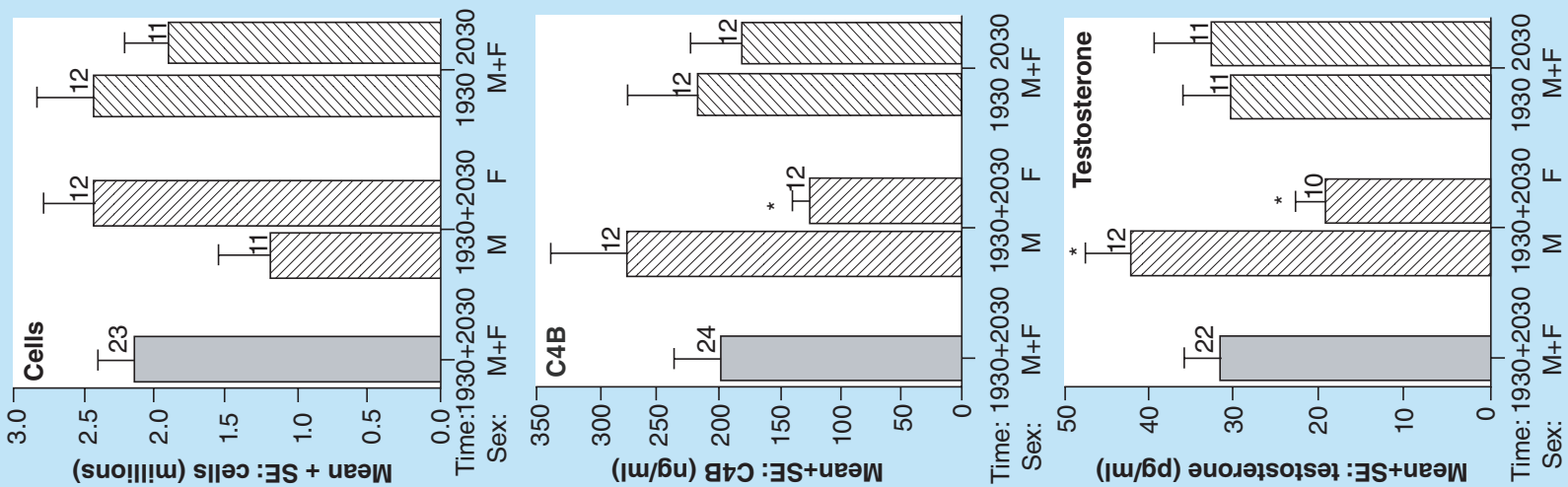
Figure 1 (see facing page). Illustration of the comparison of cell counts and concentrations of ten biomarkers that are the most likely potential candidates for use in future studies based on the detection of each analyte in at least $75 \%$ of the saliva samples collected (also see Table 5 ).

In an effort to eliminate biases, the current study included adults of similar ages (M 25-72 years, F 24-70 years) with only one of each sex in their 70s. Also, ethnicity was very similar ( $M-3$ Caucasians, 1 African, 2 Asians; F- 4 Caucasians, 2 Asians). The study is too small to determine any effect of age, ethnicity or sex.

The data produce a proof of concept that biomarkers pertinent to autism can be measured in human saliva. Further studies will need to be performed in children owing to the difficulties with saliva collection.

There were no significant differences between the two times of measurement for any analyte, albeit only $1 \mathrm{~h}$ apart, for both sexes combined. This finding illustrates that similar concentrations of the biomarkers were measured if the subjects read the instructions for saliva sample collection alone or if they later received instructions from the principal investigator. While this information allows for collection of saliva samples without the presence of the principal investigator, someone able to read and interpret the instructions nevertheless would need to be present, since many autistic individuals would need the presence of a literate individual to instruct and assist them in the proper manner of sample collection and handling.

While there were no differential counts of the types of cells present, an overall cell count was made, with numbers ranging from 0.28 to $4.40 \times 10^{6} / \mathrm{ml}$, which is consistent with the literature [77]. There was no attempt to preserve the cells, but a protease inhibitor was used to preserve the biomarkers from degradation. The saliva was frozen and stored until it was thawed for analysis. The freeze-thaw caused the cells to lyse and the samples were centrifuged prior to analysis to avoid any debris from interfering with the assay. At this point, there was no attempt to correlate the number of cells per sample with the concentrations of the biomarkers.

The saliva and the cells lining the inside of the cheeks are part of the mucosa-associated lymphoepithelial tissue. The main function of the mucosa-associated lymphoepithelial tissue is generation and dissemination of stimulated B cells (which differentiate into plasma cells and make antibodies), which subsequently require some secondary stimuli for terminal differentiation in secretory tissues. The tonsils differ from the other lymphoepithelial structures by showing considerable in situ differentiation to plasma cells. The cells in saliva include antigen-presenting cells (macrophages, dendritic cells and certain epithelial cells), and T- and B-lymphocytes. The T-lymphocytes help the B-lymphocytes transform into plasma cells due to environmental factors, which are regulatory signals.
Other regulatory signals include lymphokines, such as IFN- $\gamma$, which assist in the making of secretory antibody. Thus, the saliva and cells present are intimately involved in the body's defense mechanisms, reacting to all things that enter the mouth [78].

It was expected that the concentration of biomarkers in saliva would be less than that in blood or urine, since the saliva is considered a filtrate of the blood $[62,63]$. The extension of the standard curve in ELISAs allowed measurement of smaller concentrations. Despite this, for those eight biomarkers measured by ELISA, only five could be measured in the majority of the saliva samples: CD26, C4B, IFN- $\gamma$, GSSG and MT-2. Because GSH is very unstable and oxidizes very readily, we will not measure GSH or GSSG in our future study. Similarly, mass spectrometry was not sensitive enough to measure the hormones or two of the amino acids in saliva. The Pharmasan Laboratory measured the hormones by more sensitive means: cortisol (Electrochemiluminescent Immuno Assay), melatonin (Radio Immuno Assay) and testosterone (Enzyme-Linked Immunosorbent Assay).

We measured ten biomarkers in $\geq 74 \%$ of the saliva samples: glutamine, glutamic acid (ubiquitous), CD26 (gastrointestinal), C4B, IFN- $\gamma$ (immunologic), cortisol, melatonin, testosterone (neurologic), oxidized glutathione and Metallothionein-2 (toxicologic). Our data are consistent with the literature and provide a basis for a future study in which we plan to measure the circadian rhythm of these biomarkers. The future study will aid in producing patient-specific therapy to bring the biomarkers into synchronization and to provide homeostasis and health, lessening the symptoms of autism. This was clearly shown for melatonin [55] and the components of the methionein cycle [47].

\section{Conclusion}

In summary, the data prove the concept that saliva is an appropriate body fluid in which to measure biomarkers important to autism. We found measurable levels in $74-100 \%$ of saliva samples from 12 healthy neurotypical adults on two occasions for 10 biomarkers important to autism: glutamine, glutamic acid, CD26, C4B, IFN- $\gamma$, cortisol, melatonin, testosterone, MT-2 and GSSG, but in $<50 \%$ of samples for carnitine, GABA, IL-12, serotonin, cystine and rGSH.

\section{Future perspective}

We plan to investigate the biorhythms of the ten biomarkers in saliva for times of lowest and highest values and extent of change over $24 \mathrm{~h}$ in neurotypical individu- 
Table 6 . Concentration of biomarkers from the current study compared to the literature.

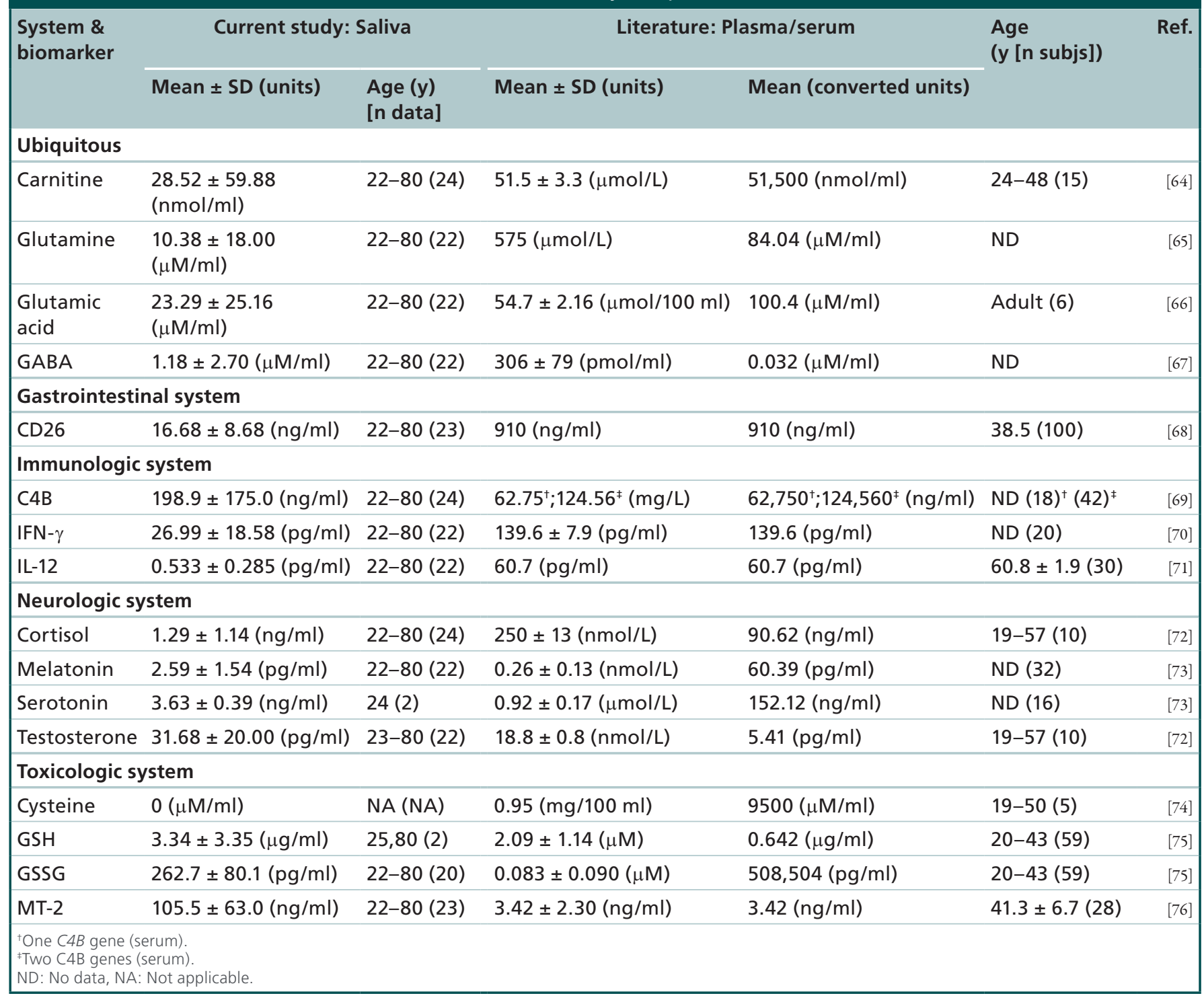

als so that 'normal' can be defined. Then the difference from normal exhibited by individuals with autism, hopefully at only one or two selected timepoints, can be used to determine a rudimentary objective measure of autism. For example, the circadian rhythms of melatonin and cortisol have been shown to be dampened in autism [25,29]. The data from the current study prove we can measure at least one biomarker from each system of the body in which autism is manifest. The planned biorhythm study should produce data, which, with the aid of discriminate and multiple regression analyses, will provide a rudimentary objective measure of autism. In biorhythm studies, measurements will be taken from saliva samples collected every $4 \mathrm{~h}$ for a total of six collections per subject in $24 \mathrm{~h}$. Concentrations of biomarkers from neurotypical individuals will be used for comparison with those from subjects with autism. The premise is that measuring the amount of difference from normal will allow the possibility of ranking the severity of autism. These data will also be correlated with existing diagnoses of autism. It is hoped that this measure will provide a patient-specific guide for therapy. After more studies, the data might also be used to help diagnose the severity of autism.

\section{Acknowledgements}

The authors are grateful to the neurotypical adults who participated by donating their saliva, and to the laboratory technicians who performed the analyses. The authors thank Ann McLellan, the quality assurance officer, whose assistance was essential to the integrity of the study.

Financial \& competing interests disclosure Edmond Enterprises LLC funded the research discussed in this article and one of the authors, HV Ratajczak, is the sole 
member of that company. The authors have no other relevant affiliations or financial involvement with any organization or entity with a financial interest in or financial conflict with the subject matter or materials discussed in the manuscript apart from those disclosed.

No writing assistance was utilized in the production of this manuscript.

\section{Ethical conduct of research}

The authors state that they have obtained appropriate institutional review board approval and have followed the principles outlined in the Declaration of Helsinki for all human or animal experimental investigations. In addition, for investigations involving human subjects, informed consent has been obtained from the participants involved.

\section{Executive summary}

- Biomarkers chosen for analysis are documented by others as being in significantly different concentrations in the blood or urine of subjects with autism compared with neurotypical controls.

- Chosen biomarkers participate in unifying concepts of the causes of autism:

- Increased vulnerability to oxidative stress

- Immune glutamatergic dysfunction

- Pineal gland malfunction

- Saliva was chosen as the body fluid because collection causes less stress than blood or urine. This results in fewer changes in concentration of the biomarkers.

- Chosen biomarkers represent systems of the body in which autism is manifest.

- Rationale for choice of biomarkers is given.

- Future studies are designed to provide a subject-specific profile of biomarkers, with a ranking of severity of the difference of concentration of each biomarker from normal, which would comprise a rudimentary objective measure of autism.

- This objective measure of autism could be a subject-specific guide for therapy.

- After more comprehensive studies, the data might also be used to diagnose the degree or progression of autism.

\section{References}

1 Rosignol DA, Frye RE. Evidence linking oxidative stress, mitochondrial dysfunction, and inflammation in the brain of individuals with autism. Front. Physiol. 5, 150 (2014).

2 Ratajczak HV. Theoretical aspects of autism: biomarkers - a review. J. Immunotox. 8(1), 80-94 (2011).

3 Jepson B. Changing the Course of Autism. Sentient Publications, Boulder CO, USA (2007).

4 Filipek PA, Juranek J, Nguyen MT, Cummings C, Gargus JJ. Relative carnitine deficiency in autism. J. Autism Dev. Disord. 34(6), 615-623 (2004).

5 Celestino-Soper PBS, Violante S, Crawford EL et al. A common X-linked inborn error of carnitine biosynthesis may be a risk factor for nondysmorphic autism. Proc. Natl Acad. Sci. USA 109(21), 7974-7981 (2012).

6 Frye RE, Rossignol DA. Treatments for biomedical abnormalities associated with autism spectrum disorder. Front. Pediatr. 2(66), 1-16 (2014).

7 Mostafa GA, AL-Ayadhi, . Reduced levels of plasma polyunsaturated fatty acids and serum carnitine in autistic children: relation to gastrointestinal manifestations. Behav. Brain Funct. 11(4), 1-10 (2015).

8 Blatt GJ, Fitzgerald CM, Guptill JT, Booker AB, Kemper TL, Bauman ML. Density and distribution of hippocampal neurotransmitter receptors in autism: an autoradiographic study. J. Autism Dev. Disord. 31(6), 537-543 (2001).

9 Marrosu F, Marrosu G, Rachel MG, Biggio G. Paradoxical reactions elicited by diazepam in children with classic autism. Funct. Neurol. 2(3), 355-361 (1987).
10 Cochrane DM, Sikoglu EM, Hodge SM et al. Relationship among glutamine, $\gamma$-aminobutyric acid, and social cognition in autism spectrum disorders. J. Child Adoles. Psychopharmacol. 25(4), 314-322 (2015).

11 Shanahan MR, Venturini AJ, Daiss JL et al. Peptide diagnostic markers for human disorders. European Patent Application, Ep 0969015 A2, 05 January 2000.

12 Bashir S, AL-Ayadhi L. Alterations in plasma dipeptidyl peptidase IV in autism: A pilot study. Neurol. Psychiat. Brain Res. 20(2), 41-44 (2014).

13 Cieslinska A, Sienkiewicz-Szlapka E, Wasilewska J et al. Influence of candidate polymorphisms on the dipeptidyl peptidase IV and $\mu$-opiod receptor genes expression in aspect of the $\beta$ - casomorphin-7 modulation functions in autism. Peptides 65, 6-11 (2015).

14 Cass H, Gringras P, March J et al. Absence of urinary opioid peptides in children with autism. Arch. Dis. Child. 93(9), 745-750 (2008).

15 Shattock P, Whiteley P. Biochemical aspects in autism spectrum disorders: updating the opioid-excess theory and presenting new opportunities for biomedical intervention. Expert Opin. Ther. Targets 6(2), 175-183 (2002).

16 Warren RP, Yonk J, Burger RW, Odell D, Warren WL. DR-positive T-cells in autism: Association with decreased plasma levels of the complement C4B protein. Neuropsychobiology 31(2), 53-57 (1995).

17 Warren RP, Singh VK, Cole P et al. Increased frequency of the null allele at the complement C4B locus in autism. Clin. Exp. Immunol. 83(3), 438-440 (1991). 
18 Kunkel SL, Ward PA, Caporale LH, Vogel C-W. The complement system. In: Immunology III. Bellanti JA (Ed.). WB Saunders Co., PA, USA, 106-116 (1985).

19 Mostafa GA, Shehab AA. The link of C4B null allele to autism and to a family history of autoimmunity in Egyptian autistic children. J. Neuroimmunol. 223(1-2), 115-119 (2010).

20 Goines PE, Croen LA, Braunschweig D et al. Increased midgestational IFN- $\gamma$, IL- 4 and IL- 5 in women bearing a child with autism: a case-control study. Molec. Autism 2(13), 1-11 (2011).

21 Ibrahim S, El-Waleely T, Zakaria N, Ismail R. A study of serum interleukin-12 in a sample of autistic children in Egypt. Egyptian J. Psychiatry 36(2), 81-87 (2015).

22 Xu N, Li X, Zhong Y. Inflammatory cytokines: potential biomarkers of immunologic dysfunction in autism spectrum disorders. Mediators Inflam. 2015, 1-10 (2015)

23 Curin JM, Terzic J, Petkovic ZB, Zekan L, Terzic IM, Susnjara IM. Lower cortisol and higher ACTH levels in individuals with autism. J. Autism Dev. Disord. 33(4), 443-448 (2003).

24 Selmaoui B, Touitou Y. Reproducibility of the circadian rhythms of serum cortisol and melatonin in healthy subjects: a study of three different 24-h cycles over six weeks. Life Sci. 73(26), 3339-3349 (2003).

25 Corbett BA, Mendoza S, Wegelin JA, Carmean V, Levine S. Variable cortisol circadian rhythms in children with autism and anticipatory stress. J. Psychiatry Neurosci. 33(3), 227-234 (2008).

26 Spratt EG, Nicholas JS, Brady KT et al. Enhanced cortisol response to stress in children in autism. J. Autism Dev. Disord. 42(1), 75-81 (2012).

27 Putnam SK, Lopata C, Thomeer ML, Volker MA, Rodgers JD. Salivary cortisol levels and diurnal patterns in children with autism spectrum disorder. J. Dev. Physical Disabil. 27(4), 453-465 (2015).

28 Corbett BA, Simon D. Adolescence, stress and cortisol in autism spectrum disorders. OA Autism 1(1), 1-6 (2013).

29 Melke J, Goubran-Botros H, Chaste P et al. Abnormal melatonin synthesis in autism spectrum disorders. Mol. Psychiatry 13(1), 90-98 (2008).

30 Novakova M, Paclt I, Ptacek R, Kuzelova H, Hajek I, Sumova A. Salivary melatonin rhythm as a marker of the circadian system in healthy children and those with attention-deficit/hyperactivity disorder. Chronobiol. Int. 28(7), 630-637 (2011).

31 Sanchez de la Peña S. The feed-sideward of cephalo-adrenal immune interactions. Chronobiologia 20(1-2), 1-52 (1993).

32 Schwichtenberg AJ, Malow BA. Melatonin treatment in children with developmental disabilities. Sleep Med. Clin. 10(2), 181-187 (2015).

33 Tilford JM, Payakachat N, Kuhlthau KA et al. Treatment for sleep problems in children with autism and caregiver spillover effects. J. Autism Dev. Disord. doi:10.1007/ s10803-015-2507-5 (2015) (Epub ahead of print).

34 Belmonte MK, Cook Jr EH, Anderson GM et al. Autism as a disorder of neural information processing: directions for research and targets for therapy. Molec. Psychiatry 9(7), 646-663 (2004).

35 Whitaker-Azmitia PM. Behavioral and cellular consequences of increasing serotonergic activity during brain development. A role in autism? Int. J. Dev. Neurosci. 23(1), 75-83 (2005),

36 Adamsen D, Ramaekers V, Ho HTB et al. Autism spectrum disorder associated with low serotonin in CSF and mutations in the SLC29A4 plasma membrane monoamine transporter (PMAT) gene. Molec. Autism 5(43), 1-11 (2014).

37 Rose'Meyer R. A review of the serotonin transporter and prenatal cortisol in the development of autism spectrum disorders. Molec. Autism 4(37), 1-16 (2013).

38 Montoya ER, Terburg D, Bos PA, van Honk J. Testosterone, cortisol, and serotonin as key regulators of social aggression: A review and theoretical perspective. Motiv. Emot. 36, 65-73 (2012).

39 Takagishi H, Takahashi T, Inukai K et al. Salivary testosterone levels and autism-spectrum quotient in adults. Neuroendocrin. Let. 31(6), 101-105 (2010).

40 Baron-Cohen S, Auyeung B, Norgaard-Pedersen B et al. Elevated fetal steroidogenic activity in autism. Molec. Psychiatry 20, 369-376 (2015).

41 Auyeung B, Baron-Cohen S, Ashwin E, Knickmeyer R, Taylor K, Hackett G. Fetal testosterone and autistic traits. Br. J. Psychology 100, 1-22 (2009),

42 Baron-Cohen $S$. The extreme male brain theory of autism. Trends Cogn. Sci. 6(6), 248-254, (2002).

43 Krahn TM, Fenton A. The extreme male brain theory of autism and the potential adverse effects for boys and girls with autism. J. Bioeth. Inquiry 9(1), 93-103 (2012).

44 Larsen FV, Lai M-C, Wagner AP, MRC AIMS Consortium, Baron-Cohen S, Holland AJ. Testing the 'Extreme Female Brain' theory of psychosis in adults with autism spectrum disorder with or without co-morbid psychosis. PLOS ONE 10(6), e0128102. (2015).

45 Tordjman S, Anderson GM, McBride PA et al. Plasma androgens in autism. J. Autism Dev. Disord. 25(3), 295-304 (1995).

46 Majewska MD, Hill M, Urbanowicz E et al. Marked elevation of adrenal steroids, especially androgens, in saliva of prepubertal autistic children. Eur. Child Adolesc. Psychiatry 23, 485-498 (2014).

47 James SJ, Cutler P, Melnyk S et al. Metabolic biomarkers of increased oxidative stress and impaired methylation capacity in children with autism. Am. J. Clin. Nutr. 80, 1611-1617 (2004).

48 Wu G, Fang Y-Z, Yang S, Lupton JR, Turner ND. Glutathione metabolism and its implications for health. J. Nutr. 134, 489-492 (2004).

49 Geier DA, Geier MR. A clinical and laboratory evaluation of methionine cycle-transsulfuration and androgen pathway markers in children with autistic disorders. Horm. Res. 66(4), 182-188 (2006).

50 Hansen RE, Roth D, Winther JR. Quantifying the global cellular thiol-disulfide status. Proc. Natl Acad. Sci. USA 106(2), 422-427 (2009).

51 Rose S, Melnyk S, Trusty TA et al. Intracellular and extracellular redox status and free radical generation in 
primary immune cells from children with autism. Autism Res. Treat. 2012, 986519 (2012). metabolism in a large autism population. Presented at: American Psychiatric Association Annual Meeting. New Orleans, LA, USA, 5-10 May 2001.

Obrenovich ME, Shamberger RJ, Lonsdale D. Altered heavy metals and transketolase found in autistic spectrum disorder. Biol. Trace Elem. Res. 144(1-3), 475-486 (2011).

54 Santos CRA, Martinho A, Quintela T, Goncalves I. Neuroprotective and neuroregenerative properties of metallothioneins. IUBMB Life 64(2), 126-135 (2012). Spectrum Disorder. Autism Research Institute, CA, USA, 182 (2012).

56 Woods AG, Wetie AGN, Sokolowska I et al. Mass specterometry as a tool for studying autism spectrum disorder. J. Molec. Psychiatry 1(6), 1-10 (2013). Woods AG. A pilot proteomic analysis of salivary biomarkers in autism spectrum disorder. Autism Res. 8(3), 338-350 (2015).

58 Wormwood KL, Aslebagh R, Channaveerappa D et al. Salivary proteomics and biomarkers in neurology and psychiatry. Proteomics Clin. Appl. doi:10.1002/ prca.201400153 (2015) (Epub ahead of print).

59 Rashid MH, Al-Jubouri RH. Assessment of serum and salivary oxidative stress biomarkers with evaluation of oral health status in a sample of autistic male children. J. Bagh College Dentistry 23(3), 56-60 (2011).

60 Monjan AA. Stress in immunologic competence: studies in animals. In: Psychoneuroimmunology. Ader R (Ed.). Academic Press, NY, USA, 185-228 (1981).

61 Corbett BA, Mendoza S, Abdullah M, Wegelin JA, Levine S Cortisol circadian rhythms and response to stress in children with autism. Psychoneuroendocrinology 31(1), 59-68 (2006).

62 Sothern RB, Roitman-Johnson B. Biological rhythms and immune function. In: Psychoneuroimmunlogy Volume 1 ( $3^{\text {rd }}$ Edition). Ader R, Felten DL, Cohen N (Eds). Academic Press, CA, USA, 445-449 (2001).

63 Sothern RB. Clinical medicine. In: Introducing Biological Rhythms. Koukkari WL, Sothern RB (Eds). Springer, NY, USA, 426-525 (2006).

64 Katrib K, Adlouni AH, Ferard G. Carnitine in human polymorphonuclear leukocytes, mononuclear cells, and platelets. Am. J. Clin. Nutr. 46, 734-735 (1987).

65 Gleeson M. Dosing and efficacy of glutamine supplementation in human exercise and sport training. J. Nutr. 138(10), S2045-S2049 (2008).
66 Stegink LD, Filer LJ Jr, Baker GL. Plasma amino acid concentrations in normal adults fed meals with added monosodium L-glutamate and aspartame. J. Nutr. 113, 1851-1860 (1983).

67 Schmidt D, Loscher W. Plasma and cerebrospinal fluid $\gamma$-aminobutyric acid in neurological disorders. J. Neurol. Neurosurg. Psychiatry 45(10), 931-935 (1982).

68 Songok EM, Osero B, Mckinnon L et al. CD26/dipeptidyl peptidase IV (CD26/DPPIV) is highly expressed in peripheral blood of HIV-1 exposed uninfected female sex workers, Virol. J. 7, 343-349 (2010).

69 Hammond A, Ollier W, Walport MJ. Effects of C4 null alleles and homoduplications on quantitative expression of C4A and C4B. Clin. Exp. Immunol. 88(1), 153-168 (1992).

70 Chodorowska G. Plasma concentrations of IFN-gamma and TNF-alpha in psoriatic patients before and after local treatment with dithranol ointment. J. Eur. Acad. Dermatol. Venereology 10(2), 147-151 (1998)

71 Wu HP, Shih CC, Lin CY, Hua CC, Chuang DY. Serial increase of IL-12 response and human leukocyte antigen-DR expression in severe sepsis survivors. 10(2), 147-151 (1998).

72 Villette JM, Bourin P, Doinel C et al. Circadian variations in plasma levels of hypophyseal adrenocortical and testicular hormones in men infected with human immunodeficiency virus. J. Clin. Endocrinol. Metab. 70(3), 572-577 (1990).

73 Rao ML, Gross G, Strebel B et al. Circadian rhythm of tryptophan, serotonin, melatonin, and pituitary hormones in schizophrenia. Biol. Psychiatry 35(3), 151-163 (1994).

74 Brigham MP, Stein WH, Moore S. The concentrations of cysteine and cystine in human blood plasma. J. Clin. Invest. 39(110, 1633-1638 (1960).

75 Jones DP, Carlson JL, Samiec PS et al. Glutathione measurement in human plasma. Evaluation of sample collection, storage and derivatization conditions for analysis of dansyl derivatives by HPLC. Clin. Chim. Acta 275(2), 175-184 (1998).

76 Milnerowicz H, Bizon A. Determination of metallothionein in biological fluids using enzyme-linked immunoassay with commercial antibody. Acta Biochim. Polonica 57(10), 99-104 (2010).

77 Xie H, Onsongo G, Popko J et al. Proteomics analysis of cells in whole saliva from oral cancer patients via value-added three-dimensional peptide fractionation and tandem mass spectrometry. Mol. Cell. Proteomics 7(3), 486-498 (2008),

78 Brandtzaeg P. Salivary immunoglobulins. In: Human Saliva : Clinical Chemistsry and Microbiology (Volume II). Tenovuo JO (Ed.). CRC Press Inc., FL, USA, 3-11(1989). 